\title{
Perceptual processing of adjacent and nonadjacent tactile nontargets
}

\author{
PAUL M. EVANS \\ Willamette University, Salem, Oregon \\ and \\ JAMES C. CRAIG and MARTHA A. RINKER \\ Indiana University, Bloomington, Indiana
}

\begin{abstract}
Previous research has shown that subjects appear unable to restrict processing to a single finger and ignore a stimulus presented to an adjacent finger. Furthermore, the evidence suggests that, at least for moving stimuli, an adjacent nontarget is fully processed to the level of incipient response activation. The present study replicated and expanded upon these original findings. The results of Experiment 1 showed that an equally large response-competition effect occurred when the nontarget was presented to adjacent and nonadjacent fingers (on the same hand). The results of Experiment 2 showed that the effects observed in Experiment 1 (and in previous studies) were also obtained with stationary stimuli. Although small, there was some indication in the results of Experiment 2 that interference may dissipate more rapidly with distance with stationary stimuli. An additional finding was that interference effects were observed in both experiments with temporal separations between the target and nontarget of up to $100 \mathrm{msec}$. In Experiment 3, target and nontarget stimuli were presented to opposite hands. Although reduced, interference was still evident with target and nontarget stimuli presented to opposite hands. Varying the physical distance between hands did not produce any change in the amount of interference. The results suggest that the focus of attention on the skin extends nearly undiminished across the fingers of one hand and is not dependent upon the physical distance between sites of stimulation.
\end{abstract}

A recent study by Evans and Craig (1991) indicated that subjects have difficulty focusing their attention on one finger and ignoring stimulation at an adjacent site. In this study, subjects were instructed to focus their attention on the left index finger and to identify the direction of movement of the stimulus presented to that finger. A second stimulus, also moving, was presented to the middle finger, adjacent to the index finger. The stimuli moved in the same direction or in opposite directions. We reasoned that if subjects could restrict their attention to the target location, the presence of the nontarget would produce little, if any, interference. If, however, subjects are unable to restrict their attention to a single finger on one hand, then the direction of movement of the adjacent nontarget might interfere with target performance in a systematic fashion. Specifically, target performance might be interfered with when the direction of movement of the nontarget was opposite to that of the target. The results showed that when the target and nontarget moved in opposite directions, accuracy was reduced and response latencies (correct trials)

This research was supported by Grant DC-00095 from the National Institutes of Health and was conducted while the first author was a visiting scholar in the Institute for the Study of Human Capabilities at Indiana University. The authors thank Roger P. Rhodes for his assistance in conducting these experiments. Reprint requests should be addressed to P. M. Evans, Department of Psychology, Willamette University, Salem, OR 97301 (e-mail: pevans@willamette.edu). were increased. On the basis of these findings, we argued that subjects could not restrict their attention to one site on the skin when an adjacent site is stimulated.

In a second study, Evans and Craig (1992) addressed the issue of the nature of the interference that occurs when adjacent sites on the skin are stimulated. For visual stimuli, where similar interfering effects have previously been demonstrated, response competition has been identified as a major source of interference. In a classic study with visual stimuli by Eriksen and Hoffman (1973), subjects were presented with a target that was flanked by distractors. There were four stimuli, but only two responses. The results showed that a significant amount of interference was observed only when the target was flanked by nontargets from the opposite response set. When the nontarget was different from the target but from the same response set, there was little interference. It appears that a visual nontarget presented in close proximity to a visual target is processed to the level of incipient response activation.

The study by Evans and Craig (1992) paralleled the work by Eriksen and Hoffman (1973). Subjects were presented with one of four tactile stimuli that moved across the finger (left, right, up, or down). Two of the stimuli were arbitrarily assigned one response, and the remaining two stimuli were assigned a second response. As in the previous study, the target location was well specified (the left index finger). Subjects were instructed to clas- 
sify the target stimulus and to ignore the stimulation on the adjacent finger. There were three trial types: The two stimuli were (1) identical (moved in the same direction) and were associated with the same response, (2) different (moved in different directions) but were associated with the same response, or (3) different and associated with different responses.

If a tactile nontarget presented to an adjacent location on the skin causes interference with the ability to identify a target simply because it is different from the target, then performance should be best when the target and nontarget are identical and worst when the target and nontarget are different. If, however, interference occurs because an adjacent nontarget is processed to the level of incipient response activation, then the amount of interference observed when the stimuli are different should depend upon the experimentally defined response mapping: Responses should be more accurate, and faster, when the stimuli are associated with the same response than when they are associated with competing responses. This is what the results unambiguously showed: Responses were consistently faster and more accurate when the stimuli were different but assigned the same response than when they were different and assigned different responses.

The sites stimulated in the studies by Evans and Craig $(1991,1992)$ were adjacent to one another, the index and middle fingers of the left hand. In the vision literature, Eriksen and Hoffman (1973) showed that the "responsecompetition effect," as it has been termed, decreased as the spatial distance between the target and nontarget increased (see also Gathercole \& Broadbent, 1987). These results have led vision researchers to propose that attention operates like a spotlight. Stimuli within the spotlight are processed in detail. Stimuli presented outside of the spotlight receive little, if any, processing (see Eriksen \& Schultz, 1979).

In the present study, we investigated whether the spotlight metaphor of attention could be extended to the tactile domain. Specifically, we investigated the interfering effect of a nontarget on a target when the stimuli were presented to adjacent and nonadjacent fingers on the same hand (Experiments 1 and 2) and to different hands (Experiment 3 ). Intuitively, it would seem to be easier to ignore a nontarget when it is presented at some distance from the target site. However, in a study of detection masking, Gilson (1969) showed that there was no difference in the amount of interference when the masking stimulus was moved from a site adjacent to the target site to a nonadjacent site. There was a decline in masking when the masking stimulus was presented to the opposite hand. Although care needs to be taken when generalizing from the results of studies of detection masking to studies of identification and categorization, it is possible that the spotlight of attention on the skin extends across an appreciable area.

In addition to investigating the effect of increasing the physical distance between the target and nontarget stimuli, we also wished to assess the generalizability of the results reported by Evans and Craig $(1991,1992)$. The stimuli used in these previous studies were horizontal and vertical bars that moved across the fingerpad. We chose to begin by investigating moving tactile stimuli because (1) movement is an inherent part of haptic exploration of objects and (2) neurophysiological evidence suggests that there are movement-sensitive neurons in primary somatosensory cortex (Costanzo \& Gardner, 1980). In Experiment 2 of the present study, we used stationary, bar-like stimuli. The question is whether the effects reported by Evans and Craig $(1991,1992)$ and those observed in Experiment 1 of the present study are restricted to moving tactile stimuli. In addition, Experiment 2 examined in more detail the effect of increasing the temporal separation between target and nontarget stimuli on the interfering effect of a nontarget. Experiment 3 investigated the effect of manipulating distance on the skin more dramatically by presenting stimuli to fingers of both hands.

\section{EXPERIMENT 1}

Before we could examine the effect of distance between target and nontarget sites, it was necessary to locate two sites that were equally effective as nontarget sites, that is, two sites that produced significant and equivalent amounts of interference on a target site located at an equal distance from the two sites. ${ }^{1}$ By analogy, a distant visual nontarget might cause less interference than a near visual nontarget not because of an increase in physical distance per se, but because the distant nontarget falls on a retinal area of low acuity and, thus, is not well perceived.

The sites we tested were the index and ring fingers as nontarget sites and the little finger as the target site. To examine the relative effectiveness of the two nontarget sites, we tested them using a target site adjacent to both nontarget sites, the middle finger. We found considerable interference at the target site from both nontarget sites but no significant difference in the amount of interference produced by the two nontarget sites. Thus, given that the ring and index fingers, as nontarget sites, produced equivalent interference on the ability to identify a target presented to the adjacent, middle finger, we can be assured that if a distance effect is observed when these two nontarget sites are used and the target site is the little finger, then this effect can be attributed to distance and not to differences in the perceptual capacities of individual fingers.

\section{Method}

Subjects. Seven subjects participated in Experiment 1, 5 females and 2 males. All were paid employees of the laboratory, and all had participated in previous experiments in the laboratory.

Apparatus. The apparatus consisted of two tactile arrays interfaced to a PDP 11/34 computer. Each tactile display was similar to that used in the Optacon (Telesensory Systems), and each consisted of 144 pins arranged in a rectangular array measuring $11 \mathrm{~mm}$ wide $\times 27 \mathrm{~mm}$ high. There were 6 columns and 24 rows of pins. Adjacent columns were separated by $2.3 \mathrm{~mm}$, and adjacent rows were separated by $1.2 \mathrm{~mm}$. When activated, the pins on the dis- 


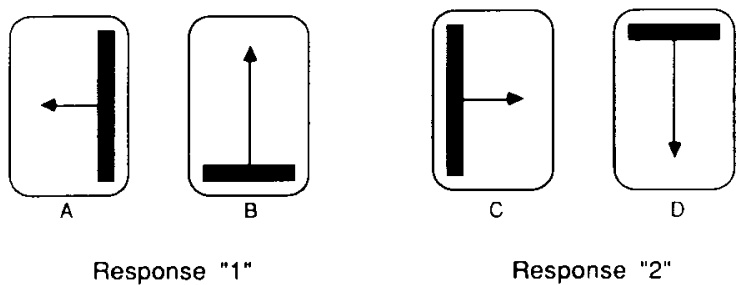

Figure 1. Illustrations of the four stimuli. Stimuli $A$ and B were assigned response "1," and Stimuli $C$ and D were ascigned response $" 2$."

play vibrated at a frequency of approximately $230 \mathrm{~Hz}$. The computer enabled spatial patterns to be generated on the tactile arrays for durations that were multiples of $4.35 \mathrm{msec}$. In addition to controlling the tactile arrays, the computer controlled a visual display and the presentation routine (used for instructions and feedback) and collected the subjects' responses (including response latencies). More detailed information about the apparatus can be found in Craig (1980).

Stimuli. There were four stimuli. Two of the stimuli (left and up) were arbitrarily assigned the response " 1 ." The remaining two stimuli (right and down) were assigned the response " 2 "' (Figure 1). The subjects received practice categorizing the stimuli before participating in the study.

Movement on each finger was simulated by shifting the location of activated pins in a column-by-column or row-by-row sequence. For example, to simulate movement across each finger from left to right, Column 1 of the tactile display was activated for $8.7 \mathrm{msec}$, followed by the activation of Column 2 for $8.7 \mathrm{msec}$, and so on. To simulate movement up the finger, for example, Rows 16, 17, and 18 were activated for $8.7 \mathrm{msec}$, followed by the activation of Rows 13, 14, and 15 for $8.7 \mathrm{msec}$, and so on. More detailed information about the stimulus conditions used to produce the perception of movement, and the procedures followed to equate the perceived intensity of the patterns on each display, can be found in Evans and Craig (1991).

Procedure. The subjects were tested with their left arms extended and with their left little fingers resting on one tactile array and their left index (or ring) fingers resting on the second tactile array. The distance between the two displays was approximately $5 \mathrm{~cm}$ in the adjacent condition and $9 \mathrm{~cm}$ in the nonadjacent condition. The subjects responded with the index and middle fingers of their right hands, which were resting on a standard keyboard. They were told that they would be presented with tactile patterns to their left little finger and left ring finger (adjacent condition) or left index finger (nonadjacent condition) and that the patterns would appear to be moving. They were instructed to focus their attention on their left little finger and to categorize the stimulus presented to that finger by pressing the button marked " 1 " or the button marked " 2 ," The subjects were explicitly instructed to ignore the stimulation on the left index finger, or the left ring finger. Accuracy was stressed, although the subjects were asked to respond as quickly as possible. The subjects were told that their responses were being timed.

A trial was initiated by the subject pressing either one of the response buttons. One second later, a moving pattern was presented to the left index finger (or left ring finger), the nontarget site. A moving pattern was also presented to the left little finger, the target site. The nontarget was presented either before the target, a 500 msec stimulus onset asynchrony (SOA) or a $100-\mathrm{msec}$ SOA, or simultaneously with the target, a 0 -msec SOA. The temporal separation between the onsets of the target and nontarget was varied on a trial-by-trial basis. Following the presentation of the two patterns, the subjects responded by pressing one of two buttons on the keyboard. A correct response was followed by the word " $\mathrm{COR}$ -
RECT" appearing on the visual display for $500 \mathrm{msec}$. An incorrect response was followed by the word "WRONG" appearing on the visual display, also for $500 \mathrm{msec}$. The visual display then went blank. By pressing either of the response buttons, the subject could then initiate the next trial. The subjects wore earphones through which white noise was presented to eliminate auditory cues produced by the tactile arrays.

Each session, which lasted approximately $30 \mathrm{~min}$, began with a block of 50 trials in which just the target was presented, followed by three blocks ( 50 trials each) with either the ring or index finger as the nontarget site, and then three blocks with the remaining site as nontarget. The order of testing the nontargets alternated daily. The subjects completed seven sessions. Target and nontarget patterns were selected at random on a trial-by-trial basis. Thus, on one fourth of the trials, the same stimulus was presented to both target and nontarget sites (stimulus-compatible condition); on one fourth of the trials, different stimuli associated with the same response were presented to the target and nontarget sites (responsecompatible condition); and on one half of the trials, different stimuli associated with different responses were presented to the target and nontarget sites (response-incompatible condition).

\section{Results and Discussion}

Both accuracy (percent correct target identification) and reaction times were recorded. ${ }^{2}$ Although the focus of the present study was on accuracy of responses, the reaction times were examined for two reasons. First, we wished to determine if there was any evidence of a speedaccuracy tradeoff. In all conditions, the opposite of a speed-accuracy tradeoff was observed, that is, as percent correct increased, reaction times decreased. Second, we wished to determine if there was any systematic relationship between response latency and distance. The reaction time data were analyzed in a three-way (nontarget location $\times$ SOA $\times$ trial type), repeated measures analysis of variance (ANOVA). The results of this analysis revealed a main effect of SOA $[F(2,12)=14.20, p<.001]$, a main effect of trial type $[F(2,12)=58.10, p<.0001]$, and an interaction between SOA and trial type $[F(4,24)=$ $3.81, p<.02]$. These reaction time results closely parallel those reported by Evans and Craig $(1991,1992)$. Specifically, reaction times decreased with increasing SOAs, and reaction times were faster in the stimulus-compatible condition than in the response-compatible condition. There was no main effect of nontarget position, nor did nontarget position interact with SOA or trial type. The threeway interaction was also not significant $(p>.05$ in all cases). In short, there was no evidence from the reaction time data that the position of the nontarget affected the speed of the subjects' responses.

The percent correct results were analyzed in a threeway, repeated measures ANOVA. The results of this analysis revealed a main effect of trial type $[F(2,12)=37.00$, $p<.0001]$, a main effect of SOA $[F(2,12)=10.61, p<$ .011 , and an interaction between SOA and trial type $[F(4,24)=22.98, p<.0001]$. There was no main effect of nontarget location. Following this initial analysis, planned comparisons ( $t$ tests) were conducted.

Figure 2 shows the percentages of correct responses for the three trial types, as a function of SOA, for the adjacent and nonadjacent conditions. The top panel shows the 


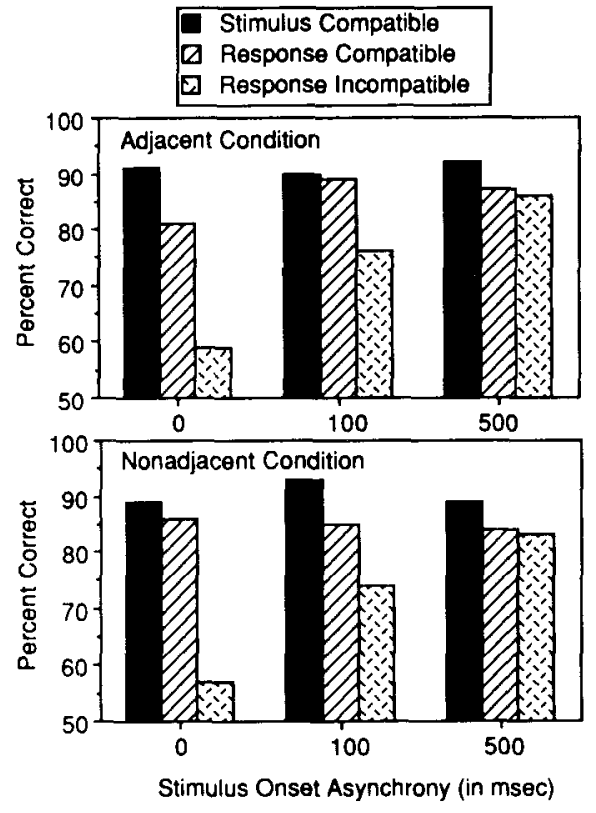

Figure 2. Percent correct identification for three trial types, stimulus compatible, response compatible, and response incompatible, as a function of the time between the onsets of the target and nontarget stimuli (SOA). The target and nontarget stimuli were moving. The upper panel presents the data from the condition in which the target site was the little finger and the nontarget site was the ring finger (adjacent condition). The lower panel presents the data from the condition in which the target site was the little finger and the nontarget site was the index finger (nonadjacent condition).

results for the adjacent condition. At a 0 -msec SOA, performance was best in the stimulus-compatible condition $(91 \%)$ and worst in the response-incompatible condition (59\%). Performance in the response-compatible condition $(81 \%)$ was worse than that obtained in the stimuluscompatible condition $(p<.05)$. The reduction in performance in the response-incompatible condition was also significant $(p<.05)$. A similar pattern of results was obtained at a 100-msec SOA. Performance in the response-incompatible condition was significantly poorer than that in the response-compatible condition $(p<.05)$. At a $500-\mathrm{msec}$ SOA, performance was statistically equivalent for the three trial types (average $=88 \%$ ). Singlepattern performance averaged $93 \%$.

The bottom panel of Figure 2 shows the percentages of correct responses for the three trial types as a function of SOA when the nontarget location was the index finger (nonadjacent condition). The results are very similar to those illustrated in the top panel (adjacent condition). At a 0-msec SOA, performance was best in the stimuluscompatible condition (89\%) and worst in the responseincompatible condition (57\%). Performance in the response-compatible condition (86\%) was statistically equivalent to that obtained in the stimulus-compatible condition $(p>.05)$. The reduction in performance in the response-incompatible condition from that obtained in the response-compatible condition was significant $(p<.05)$. At a 100-msec SOA, performance was best in the stimulus- compatible condition (93\%) and worst in the responseincompatible condition $(74 \%, p<.05)$. Performance in the response-incompatible condition was significantly poorer than that obtained in the response-compatible condition $(p<.05)$. At a 500-msec SOA, performance was statistically equivalent for all three trial types (average $=$ $85 \%, p>.05$ ).

The similarity of the results in the adjacent and nonadjacent conditions suggests that subjects cannot restrict processing to one stimulus on the hand even when the second stimulus is presented at some distance from the target site. Moreover, the fact that the magnitude of the interference effect was approximately the same in both the adjacent and nonadjacent conditions implies that a distant nontarget presented to the same hand as a target is treated no differently by the processing system than an adjacent nontarget. Is it possible, however, that a ceiling effect may have obscured a potential distance effect? In other words, is it possible that the nonadjacent nontarget was such a potent distractor that no additional interference was possible in the adjacent condition? The amount of interference was certainly quite large. If a ceiling effect had obscured a distance effect, then reducing the effectiveness of the distractor should have led to a greater reduction in the amount of interference in the nonadjacent condition than in the adjacent condition. Increasing the SOA from 0 to 100 msec reduced the effectiveness of the nonadjacent distractor but did not result in a greater reduction in the amount of interference than in the adjacent condition. The evidence suggests that the absence of a distance effect was not the result of a ceiling effect.

The results of Experiment 1 replicate and extend those reported by Evans and Craig (1992). First, there was a significant response-competition effect indicating that the nontarget stimulus was being processed to the level of incipient response activation. The response-competition effect appears to be a particularly robust finding. Second, the effect did not depend on the physical distance between the target and nontarget. A significant and statistically equivalent response-competition effect was observed in both the adjacent and nonadjacent conditions. It appears, at least for moving stimuli, that subjects cannot restrict processing to one stimulus on the hand.

\section{EXPERIMENT 2}

In Experiment 2, we examined the interference effects observed in Experiment 1 using different stimuli. We wished to determine the extent to which the interference effect in general, and more specifically the effect of distance, is dependent upon the nature of the stimuli. In the vision literature, where it has been shown that the interfering effect diminishes with increasing distance between a target and nontarget, the stimuli are typically stationary, spatial patterns. Using moving stimuli, Driver and Baylis (1991) showed no decrease in interference with increasing distance; however, Kramer, Tham, and Yeh (1991) have been unable to replicate these findings. It is possible, though, that the absence of a distance effect in 
Experiment 1 was due, in part, to the use of moving stimuli. In Experiment 2, we used stationary stimuli that differed from each other in their locations on the fingerpad. In addition to varying the nature of the stimuli, additional SOAs were tested to examine the temporal course of the interference effect.

\section{Method}

Subjects. Five subjects participated in Experiment 2, 4 females and 1 male. All subjects had participated in Experiment 1.

Apparatus. The same apparatus used in Experiment 1 was used in Experiment 2.

Procedure. The major differences in procedures between Experiments 1 and 2 were (1) the nature of the stimuli used, and (2) the SOAs between nontarget and target stimuli. In Experiment 2, two vertical bars, one on the left (column 1) and one on the right (column 5) of the display, and two horizontal bars, one at the top and one at the bottom of the array, composed the four stimuli. These stimuli were stationary. Each stimulus consisted of 18 pins and was presented for $13 \mathrm{msec}$. The left and top stimuli were assigned response " 1 ," and the right and bottom stimuli were assigned response " 2 ." Five SOAs were tested, 0, 52, 100, 150, and $500 \mathrm{msec}$. The subjects received two practice sessions in categorizing these stimuli before participating in the study. As in Experiment 1, each testing session began with a block of 50 single-pattern trials. This block of trials was followed by three blocks of trials with either the ring or index finger as the nontarget site, then another block of single-pattern trials followed by three blocks with the remaining site as nontarget. The subjects completed a total of five sessions.

In Experiment 2, we first examined the index and ring fingers to see if they were equally effective as nontarget sites with the stationary stimuli. The test site was the middle finger. The results from 5 subjects tested for two sessions showed that, as had been the case with the moving stimuli (Experiment 1), the two nontarget sites produced equivalent amounts of interference.

\section{Results and Discussion}

The results, percent correct, were analyzed, as in Experiment 1 , in a three-way (nontarget location $\times$ SOA $x$ trial type), repeated measures ANOVA. The results of this analysis showed a main effect of trial type $[F(2,8)=15.49, p<.002]$ and a main effect of SOA $[F(4,16)=5.45, p<.01]$. There was no main effect of nontarget location, and no interaction between trial type and nontarget position ( $p>.05$ in both cases). There was an interaction between nontarget position and SOA $[F(4,16)=8.66, p<.001]$, an interaction between trial type and SOA $[F(8,32)=9.29, p<.0001]$, and a significant three-way interaction $[F(8,32)=2.28, p<.05]$. Having established these reliable main effects and interactions, more specific, planned comparisons were conducted.

Figure 3 shows the percentages of correct responses for the three trial types, as a function of SOA, for the adjacent and nonadjacent conditions. The top panel shows the results for the adjacent condition. The results are very similar to those observed in Experiment 1, namely, at a 0 -msec SOA, performance was best in the stimuluscompatible condition (86\%) and worst in the responseincompatible condition (68\%). This difference was significant $(p<.05)$. In addition, performance in the responseincompatible condition was significantly worse than that
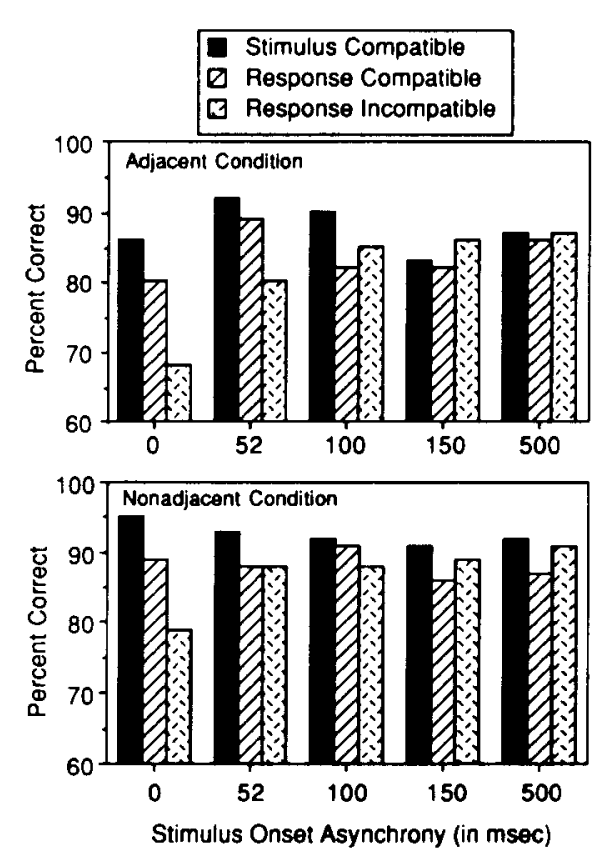

Figure 3. Percent correct identification for three trial types as a function of SOA. The stimuli were stationary. The upper panel presents the data from the condition in which the target site was the little finger and the nontarget site was the ring finger (adjacent condition). The lower panel presents the data from the condition in which the target site was the little finger and the nontarget site was the index finger (nonadjacent condition).

observed in the response-compatible condition $(80 \%, p<$ $.05)$.

The bottom panel of Figure 3 shows the results when the nontarget was presented to a nonadjacent site. The results are similar to those seen in the adjacent condition (top panel). At a 0-msec SOA, performance was best in the stimulus-compatible condition and significantly worse in the response-incompatible condition $(p<.05)$, with performance in the response-compatible condition intermediate between the other two conditions. For target and nontarget stimuli presented simultaneously, the amount of interference appears to be independent of the distance between the target and nontarget sites (as observed in Experiment 1) and independent of the nature of the stimuli.

There was some indication of a distance effect as the temporal separation between target and nontarget increased. Specifically, at a $52-\mathrm{msec}$ SOA, there was still a significant difference between the response-compatible and response-incompatible conditions for adjacent stimuli; however, for nonadjacent stimuli at the same SOA, there was no difference among trial types. By a 100-msec SOA, there was no significant interference effect in either adjacent or nonadjacent conditions.

As in Experiment 1, the reaction time data were analyzed in a three-way (nontarget location $\times$ SOA $\times$ trial type), repeated measures ANOVA. The results of this analysis showed a main effect of SOA $[F(4,16)=22.39$, $p<.0001]$, a main effect of trial type $[F(2,8)=55.13$, 
$p<.0001]$, and an interaction between SOA and trial type $[F(8,32)=3.51, p<.01]$. There was no main effect of nontarget position. All of these results parallel the results reported in Experiment 1; however, unlike the results of Experiment 1, there was a significant interaction between nontarget position and trial type $[F(2,8)=6.42$, $p<.05]$. Consistent with the percent correct results, the amount of interference produced by an incompatible, nontarget stimulus varied as a function of the position of the nontarget. Specifically, presenting the incompatible nontarget stimulus resulted in an increase in reaction times of $38 \mathrm{msec}$ at a 0 -msec SOA and $30 \mathrm{msec}$ at a $52-\mathrm{msec}$ SOA (as compared with presenting a compatible nontarget) in the adjacent condition. For the nonadjacent condition, the comparable increases were smaller, 23 and 14 $\mathrm{msec}$, suggesting a decline in interference as distance increased.

The results at a 52-msec SOA are the only significant effect of distance on percent correct in Experiments 1 and 2 , and, although this effect is supported by a comparably small reaction time effect, one needs to proceed cautiously in interpreting the results. It is possible that the distance effect dissipates more rapidly with increasing SOA for nonadjacent than adjacent stimuli. However, the magnitude of the reduction in percent correct at a 0 -msec SOA is approximately the same for the two distance conditions, and the overall similarity of the adjacent and nonadjacent results suggests that if there is an effect of distance, it is not particularly compelling. For the present, it may be best to conclude that across the hand, there is little evidence for a major effect of distance.

\section{EXPERIMENT 3}

It may not be too surprising that moving the nontarget from an adjacent to a nonadjacent finger on the same hand has little effect on the amount of interference. After all, the fingers tend to work together when exploring an object haptically, and the distance between fingers varies as the object is manipulated. The results from Experiments 1 and 2 suggest that all stimuli presented to one hand are processed to some degree and that the degree of processing is independent of the distance between the target and nontarget. If, however, the target and nontarget stimuli were presented to separate hands, less interference might be expected. The fingers of different hands are typically less likely to come into contact with the same object or parts of an object than fingers on the same hand.

Essick and Whitsel (1988) found some evidence for interference with a moving target stimulus presented to the left hand and a nontarget stimulus presented to the left shoulder. They found no interference when the nontarget was presented to the right hand; however, they also found no interference when the nontarget was placed closer to the target, so it is difficult to draw a definite conclusion from this study about the effect of presenting target and nontarget stimuli to quite distant sites on the skin. As noted before, in a study on masking in which subjects attempted to detect a target on one location on the fingers and ignore a masking stimulus presented to another location, more interference occurred when stimuli were presented to the same hand than when they were presented to different hands (Gilson, 1969).

In Experiment 3, we assessed the extent to which presenting a nontarget to a different hand reduced or eliminated its interfering effect on a target within the context of the present paradigm. In a previous study in which only two rather than four stimuli were tested (Evans \& Craig, 1991), the incompatible nontarget produced no interference effect. Preliminary observations in our laboratory indicated that increasing the number of stimuli (from two to four) produced a greater interference effect, although less interference was seen when the target and nontarget were presented to different hands than when they were presented to the same hand.

In addition to assessing the effect of increasing the anatomical distance between target and nontarget, presenting stimuli to different hands also enables us to manipulate the physical distance between the stimulators while leaving the sites of stimulation unchanged. The hands can be placed close together or far apart. It is possible, for example, that the magnitude of the interfering effect of a nontarget will depend not only on whether stimuli are presented to the same hand or different hands but also on the location of the hands in space. Subjects may be better able to focus attention on one site of stimulation when the nontarget is both anatomically and physically distant than when it is near the target site. To this end, subjects were presented with target and nontarget stimuli to the same hand (ipsilateral condition), to both hands placed close together (bilateral-near condition), and to both hands placed at some distance from each other (bilateral-far condition).

\section{Method}

Subjects. Twelve subjects participated in Experiment 3. Two of these subjects had participated in Experiment 1, and 1 of these 2 had also participated in Experiment 2. The additional subjects had participated in related experiments in the laboratory.

Apparatus. The apparatus used in Experiments 1 and 2 was used in Experiment 3.

Procedure. The procedural details of Experiment 3 were essentially identical to those of Experiment 2 . The differences were as follows: (1) Only two SOA values were tested, 0 and $100 \mathrm{msec}$, and (2) the target and nontarget stimuli were presented to fingers of the same hand (ipsilateral presentation) or to fingers of both hands (bilateral presentation). Specifically, in the ipsilateral condition, the target site was the little finger of the left hand and the nontarget site was the adjacent ring finger. The distance between the two displays measured from the center of each display was approximately $5 \mathrm{~cm}$. In one bilateral condition (bilateral-near), the target site was the little finger of the left hand and the nontarget site was the ring finger of the right hand. In this case, distance between the two displays was approximately $10 \mathrm{~cm}$, comparable to the distance between the two displays in the nonadjacent conditions of Experiments 1 and 2. In a second bilateral condition (bilateral-far), the physical distance between fingers was increased by separating the displays by $42 \mathrm{~cm}$. The target and nontarget sites remained the same. The subjects responded via foot pedals rather than a keyboard. 
Six subjects were presented with moving stimuli, and an additional 6 subjects were presented with static stimuli. Three conditions were tested: ipsilateral, bilateral-near, and bilateral-far. Each condition began with a block of 50 single-pattern trials. This block was followed by three blocks of trials in which both a target and nontarget stimulus were presented. The conditions were tested in order, ipsilateral, bilateral-near, and bilateral-far. Two of the three conditions were presented in a typical session. For example, if the ipsilateral and bilateral-near conditions were tested in the first session, then the bilateral-far and ipsilateral conditions were tested in the second session. The subjects completed each condition six times, with the exception of 1 of the subjects in the static group who completed each condition only five times. Because of mechanical difficulties with the foot pedals, reaction times were not analyzed.

\section{Results and Discussion}

Because different subjects were tested with the moving and static stimuli, separate three-way, repeated measures ANOVAS were conducted for each data set, that is, for the moving stimuli and for the stationary stimuli. The results for the moving stimuli are presented first.

Moving stimuli. The results of the ANOVA revealed a main effect of nontarget location $[F(2,10)=13.95, p<$ $.01]$, a main effect of trial type $[F(2,10)=9.15, p<$ $.01]$, and an interaction between nontarget location and trial type $[F(4,20)=4.27, p<.05]$. The results are shown in Figure 4.

The top panel of Figure 4 shows the results for the ipsilateral condition. The results are very similar to those obtained in the comparable condition of Experiment 1. Specifically, at a 0-msec SOA, performance was best in the stimulus-compatible condition (86\%) and worst in the response-incompatible condition $(70 \%)$. At a $100-\mathrm{msec}$ SOA, there was still an interference effect, although the amount of interference was reduced.

The middle panel of Figure 4 shows the results for the bilateral-near condition. As observed before, performance at a 0-msec SOA was best in the stimulus-compatible condition ( $90 \%$ ) and worst in the response-incompatible condition $(83 \%)$. Compared with the ipsilateral condition, the amount of interference was, however, reduced by more than half $(15 \%$ vs. $7 \%)$.

The bottom panel of Figure 4 shows the results for the bilateral-far condition when the displays were placed $42 \mathrm{~cm}$ apart. The results are very similar to those shown in the middle panel. Performance was best in the stimuluscompatible condition (89\%) and worst in the responseincompatible condition (83\%). The amount of interference was approximately the same as that observed when the displays, and thus the target and nontarget sites, were physically closer (6\% vs. $7 \%$ ).

Static stimuli. The results of the ANOVA showed a significant main effect of trial type $[F(2,10)=17.41, p<$ $.001]$, a significant interaction between SOA and trial type $[F(2,10)=4.51, p<.05]$, and a significant interaction between nontarget position and trial type $[F(4,20)=3.95$, $p<.05]$. The results are shown in Figure 5 .

The results shown in Figure 5 are very similar to those shown in Figure 4, with the exception that the overall amount of interference with the static stimuli was reduced
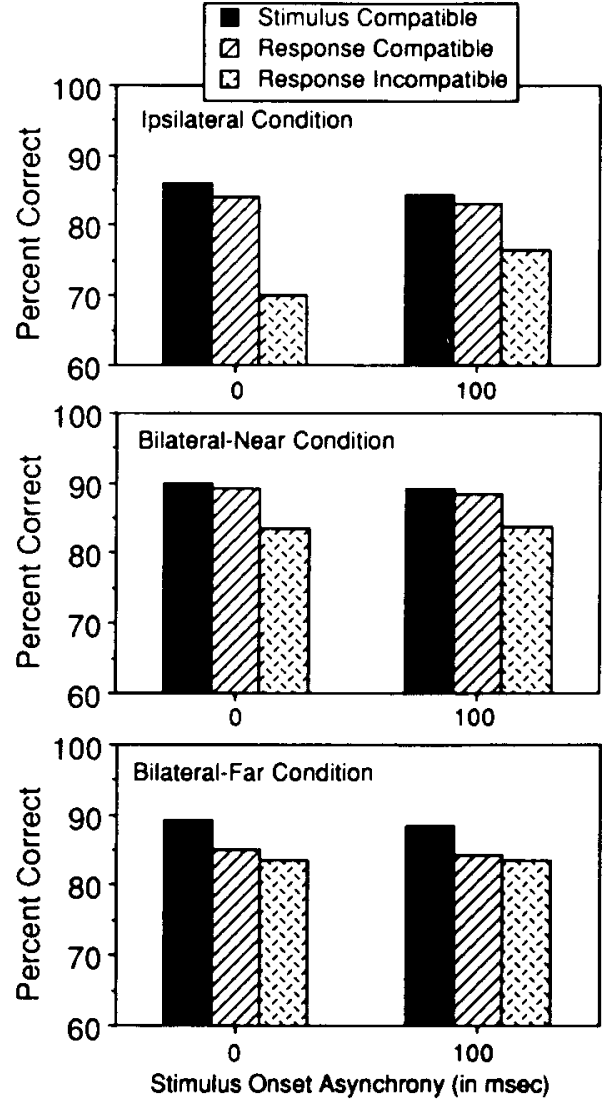

Figure 4. Percent correct identification for three trial types as a function of SOA. The stimuli were moving. The results from three different conditions are presented: ipsilateral, two sites on the same hand; bilateral-near, two sites on opposite hands with the hands adjacent to one another; and bilateral-far, two sites on opposite hands with the hands separated by $42 \mathrm{~cm}$ from one another.

from that observed with the moving stimuli. A similar reduction in the amount of interference was observed between Experiment 1 (moving stimuli) and Experiment 2 (static stimuli). However, in spite of a reduction in the amount of interference, there was still greater interference in the ipsilateral condition than in the bilateral conditions. Furthermore, and as observed with the moving stimuli, the magnitude of the interference effect in the bilateral conditions did not decrease as the physical distance between the displays was increased. Indeed, at a 100 -msec SOA, there was no interference in the bilateralnear condition and $6 \%$ interference in the bilateral-far condition.

To determine if there was any difference between the bilateral-near and bilateral-far results, a three-way ANOVA was conducted on the bilateral data. For the moving stimuli, the results of this analysis revealed a significant effect of trial type $[F(1,5)=7.96, p<.01]$ and a significant effect of position $[F(1,5)=7.91, p<.05]$. For the static stimuli, the analysis revealed a significant effect of trial type $[F(1,5)=13.31, p<.01]$ and a sig- 

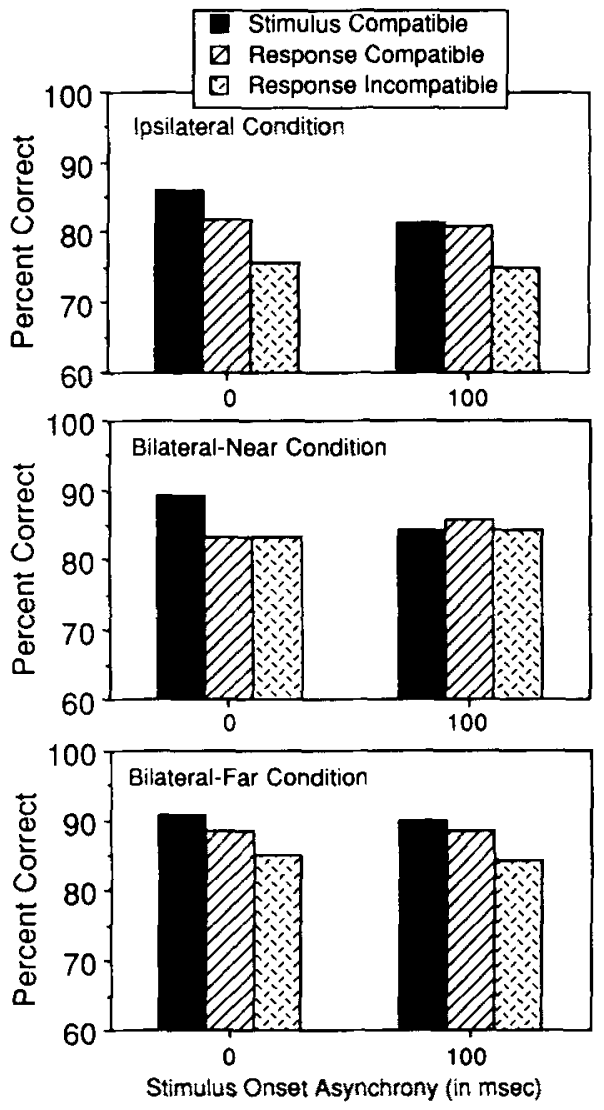

Figure 5. Percent correct identification for three trial types as a function of SOA. The stimuli were stationary. The results from three different conditions are presented: ipsilateral, bilateral-near, and bilateral-far.

nificant three-way interaction $[F(2,10)=9.04, p<.01]$. The significant main effect of trial type, for both the moving and nonmoving stimuli, reflects the finding that performance was typically best in the stimulus-compatible condition and worst in the response-incompatible condition, both at 0 - and $100-\mathrm{msec}$ SOAs. The fact that there was no interaction between trial type and hand position is evidence that the magnitude of the interference effect did not depend on the physical distance between the stimuli.

The fact that there was no difference between the bilateral-near and bilateral-far conditions parallels earlier bilateral results (Craig, 1985). The task in this earlier study was to attend to two sites on the skin and combine the information from the two sites. Performance in bilateral conditions was superior to ipsilateral conditions, but performance was unaffected by placing the hands either adjacent to one another or far from one another. In short, and as observed in the present study, performance was unaffected by physical distance between the two hands.

\section{GENERAL DISCUSSION}

The results of the present study replicate and extend earlier results by Evans and Craig $(1991,1992)$. First, in all three experiments, there was a significant amount of interference when the target and nontarget stimuli were associated with different responses. The responsecompetition effect originally advanced by Eriksen and his colleagues (Eriksen \& Hoffman, 1973; Eriksen \& Schultz, 1979) appears to be a particularly robust finding, one that is not restricted to visually presented stimuli. Second, there was little effect of increasing the distance between the target and nontarget stimuli as long as the stimuli were presented to the same hand (Experiments 1 and 2). Although stimuli may be better perceived on some fingers than on others, it appears that once registered, stimuli presented to the same hand are fully processed to the level of "incipient response activation" (Eriksen \& Schultz, 1979 , p. 259), regardless of whether subjects are attempting to focus their attention on that site and regardless of whether the target and nontarget stimuli are close together on the same hand. The absence of a compelling distance effect when stimuli are presented to the same hand is consistent with the finding by Eriksen and his colleagues that distance had little effect as long as the visual stimuli were presented within approximately $1^{\circ}$ of visual angle. Third, subjects do appear to be better able to focus their attention on a target when a nontarget is presented to a different hand than when it is presented to the same hand (Experiment 3).

The results of the present study indicate that information about the location of a specific tactile stimulus may not be intrinsically encoded along with information about the identity of the stimulus, at least when stimuli are presented to one hand. This suggestion is consistent with the results of a number of studies in the vision literature that suggest that information used to identify a stimulus can become separated from information about the location of the stimulus (Estes, Allmeyer, \& Reder, 1976; Mozer, 1989; Treisman \& Gelade, 1980). What is surprising, perhaps, is that we might have expected the amount of spatial uncertainty to decrease as the physical distance between the stimuli was increased. However, unlike the fixed, location-invariant surface of the retina, the sensory surface of the fingers can be manipulated and the physical distance between adjacent and nonadjacent fingers on the same hand can be increased and decreased at will. Thus, increasing the physical distance between tactile stimuli (by presenting them to nonadjacent fingers) may not be akin to increasing the physical distance between visually presented stimuli. The evidence suggests that to a subject identifying spatial patterns, a specific finger on one hand may (perceptually) be as distant from an adjacent finger as it is from a nonadjacent finger on the same hand.

The present results raise the issue of what is meant by the term distance when applied to stimuli presented to the 
skin. The term distance can be used in at least three ways. It can be used to refer to the physical distance between stimuli, to the perceived distance between stimuli, and to the cortical distance between the initial representation of stimuli (Jenkins, Merzenich, Ochs, Allard, \& GuícRobles, 1990). The relationship among these three applications of the term distance appears to be a complicated one (see Geldard, 1975; Geldard \& Sherrick, 1972; Green, 1982; Helson \& King, 1931), and a detailed examination of this relationship is beyond the scope of the present study. It may be important to note, however, that this issue is not restricted to tactually presented stimuli. In a widely known study, Treisman and Gelade (1980) demonstrated that spatially separated attributes of visual stimuli may be combined erroneously and be perceived as if they had been presented as part of one stimulus. More recently, Baylis and Driver (1992) have shown that visually presented targets and nontargets that are presented at some distance from one another but that share an attribute (such as color) can yield a greater interference effect than stimuli that are presented closer together in space but that do not share the same attribute. It remains to be seen whether similar effects can be demonstrated on the skin.

Moving the target to a separate hand reduced the amount of interference for both moving and stationary stimuli. The reduction in interference does not seem to be due to an increase in the physical distance between the stimulators. Increasing the separation from 5 to $9 \mathrm{~cm}$ in Experiments 1 and 2 produced at best only a marginal decline in the amount of interference. Increasing the separation by only $1 \mathrm{~cm}$ (to $10 \mathrm{~cm}$ ), the separation in the bilateralnear condition in Experiment 3, would be unlikely to produce the decline in interference that was seen. Moreover, increasing the separation to $42 \mathrm{~cm}$ produced no further reduction in the amount of interference. The bilateral results suggest that the spotlight metaphor of attention for the skin may not be entirely appropriate. Factors other than distance between the target and nontarget may play an important role in determining the amount of interference. A similar suggestion for visual stimuli has recently been made by Baylis and Driver (1992). In haptic exploration, the two hands may be used either together or separately, at very close physical separations or at quite some distance from one another. This mobility of the sensory surface relative to the physical environment most likely affects the nature of attentional mechanisms on the skin. To test the spotlight metaphor in a manner more similar to that seen with visual stimuli, one could examine a sensory surface such as the forearm or thigh and vary the distance between target and nontarget. When the forearm or thigh is moved, the points of stimulation do not change their physical separation from one another.

In the present experiments, the subjects were instructed to attend to only one location and ignore stimulation at a second, nontarget, location. The fact that the nontarget stimulus affected processing of the target stimulus can be taken as evidence that subjects are processing patterns at two locations. Several previous studies have required subjects to attempt to attend to several sites simultaneously (Craig, 1985; Lederman \& Klatzky, 1987; Sathian \& Burton, 1991). In one of these studies using stimuli very similar to those used in the present study, subjects attempted to combine information from two sites (Craig, 1985). Subjects were able to combine patterns presented to two fingers, but performance was better when the two patterns were combined and presented to a single finger. These results suggested that subjects could divide their attention between two sites, but at the cost of some loss of information. The present results are consistent with this view of attending to several locations on the same hand.

Subjects are better able to combine bilateral patterns than ipsilateral patterns presented to adjacent fingers on the same hand (Craig, 1985). This result suggests that greater interference should be seen between target and nontarget stimuli presented bilaterally than ipsilaterally. The fact that the opposite result is found points to a difference between tasks in which subjects are required to focus attention on one location and attempt to ignore other sites (the present experiments) and tasks in which subjects attempt to divide attention between two sites and combine information from the sites (Craig, 1985). The present results are consistent with previous results if one views the two hands as separately controlled resources. For bilateral stimuli, subjects show greater flexibility in complying with task requirements and seem better able to either combine or ignore stimuli. For ipsilateral stimuli, subjects have less flexibility over allocating resources and are less able to comply with the changing task requirements.

The effects reported in this study are quite large. Under some conditions, correct responding was reduced from better than $90 \%$ correct to less than $10 \%$ above chance. With such potential for confusion, one might well ask how individuals are ever able to identify complex objects haptically. As Klatzky, Lederman, and Metzger (1985) have shown, blindfolded subjects are extremely accurate in identifying common three-dimensional objects. The authors also report, however, that typical response latencies were in the range of 1-2 sec. The effects reported in the present study were observed over a much more restricted temporal range. Typically, we have observed relatively little interference when the temporal separation between the target and nontarget exceeds $150-200 \mathrm{msec}$. Extending the sampling of features over a time period of 1-2 sec would most likely lead to little interference of the type reported here. Also, haptic exploration often involves purposeful, self-generated finger movements (see Lederman \& Klatzky, 1990). Such movements permit a person to direct the fingers to relevant parts of an object and to return to these parts to confirm the presence (or absence) of a feature. These confirmatory motions would certainly be expected to reduce the likelihood of errors.

Although the focus of the present study was on response competition as a function of the spatial distance between a target and nontarget, and on the generalizability of the 
findings, two additional findings are worthy of discussion. First, single-pattern performance was typically better than that obtained when identical targets and nontargets were presented. A similar effect has been reported in the vision literature (Bjork \& Murray, 1977; Eriksen \& Schultz, 1979; Mozer, 1989). One interpretation of this finding is that the initial encoding involves the complete stimulus array as a global unit. Decisions about specific attributes of the array (a specific stimulus in a specific location) may require additional time and processing resources. When only one stimulus is presented, the complete stimulus array is the stimulus to be responded to. Second, responses on stimulus-compatible trials were typically more accurate than responses on response-compatible trials. The effect was not large, but was observed in all experiments (and was observed earlier by Evans \& Craig, 1992). The responses for these two trial types were the same; only the stimuli were different. In this sense, the finding parallels that reported by a number of investigators that "same" responses to identical stimuli are typically more accurate and faster than correct "different" responses (Bamber, 1969; Krueger, 1970, 1978; Nickerson, 1965; Proctor, 1981). A number of explanations of the fast "same" effect have been advanced in the literature. Because the present study did not involve discrimination judgments, it would be inappropriate to speculate at this point about a fast "same" effect for tactually presented stimuli. The results do, however, suggest that, as with visually presented stimuli, there is something special about identity (Eriksen, O'Hara, \& Eriksen, 1982; Evans \& Smith, 1988; Smith \& Evans, 1989). Further empirical research is called for to investigate the locus of this effect with tactile stimuli.

\section{REFERENCES}

BAmber, D. (1969). Reaction times and error rates for "same" - "different" judgments of multidimensional stimuli. Perception \& Psychophysics, 6, 169-174.

BAYLIS, G. C., DRIVER, J. (1992). Visual parsing and response competition: The effect of grouping factors. Perception \& Psychophysics, 51, 145-162.

Bjork, E. L., MurRay, J. T. (1977). On the nature of input channels in visual processing. Psychological Review, 84, 472-484.

Costanzo, R. M., GARDNer, E. P. (1980). A quantitative analysis of responses of direction-sensitive neurons in the somatosensory cortex of alert monkeys. Journal of Neurophysiology, 43, 1319-1341.

Craig, J. C. (1980). Modes of vibrotactile pattern generation. Journal of Experimental Psychology: Human Perception \& Performance, 6, 151-166.

CralG, J. C. (1985). Attending to two fingers: Two hands are better than one. Perception \& Psychophysics, 38, 496-511.

DRIVER, J., \& BAYLIS, G. C. (1991). Target-distractor separation and feature integration in visual attention to letters. Acta Psychologia, 76, 101-119.

Eriksen, C. W., \& Hoffman, J. E. (1973). The extent of processing noise elements during selective encoding from visual displays. Perception \& Psychophysics, 14, 155-160.

Eriksen, C. W., O'HARA, W. P., ERIKSEN, B. (1982). Response competition effects in same-different judgments. Perception \& Psychophysics, 32, 261-270.
ERIKSEN, C. W., \& SChultZ, D. W. (1979). Information processing in visual search: A continuous flow conception and experimental results. Perception \& Psychophysics, 25, 249-263.

Essick, G. K., WHITSEL, B. L. (1988). The capacity of human subjects to process directional information provided at two skin sites. Somatosensory \& Motor Research, 6, 1-20.

Estes, W. K., Allmeyer, D. H., \& Reder, S. M. (1976). Serial position functions for letter identification at brief and extended exposure durations. Perception \& Psychophysics, 19, 1-15.

Evans, P. M., \& Cralg, J. C. (1991). Tactile attention and the perception of moving tactile stimuli. Perception \& Psychophysics, 49, 355-364.

Evans, P. M., \& Craig, J. C. (1992). Response competition: A major source of interference in a tactile identification task. Perception \& Psychophysics, 51, 199-206.

Evans, P. M., Smith, L. B. (1988). The development of identity as a privileged relation in classification: When very similar is just not similar enough. Cognitive Development, 3, 265-284.

Gathercole, S. E., \& Broadbent, D. E. (1987). Spatial factors in visual attention: Some compensatory effects of location and time of arrival of nontargets. Perception, 16, 433-443.

Geldard, F. A. (1975). Sensory saltation. New York: Academic Press. Geldard, F. A., \& SHerrick, C. E. (1972). The cutaneous "rabbit": A perceptual illusion. Science, 178, 178-179.

GiLsoN, R. D. (1969). Vibrotactile masking: Some spatial and temporal aspects. Perception \& Psychophysics, 5, 176-180.

GreEN, B. G. (1982). The perception of distance and location for dual tactile pressures. Perception \& Psychophysics, 31, 315-323.

Helson, H., \& King, S. M. (1931). The tau-effect. An example of psychological relativity. Joumal of Experimental Psychology, 14, 202-218

Jenkins, W. M., Merzenich, M. M., Ochs, M. T., Allard, T., \& Guic-Robles, E. (1990). Functional reorganization of primary somatosensory cortex in adult owl monkeys after behaviorally controlled tactile stimulation. Journal of Neurophysiology, 63, 82104.

Klatzky, R. L., Lederman, S. J., \& Metzger, V. A. (1985). Identifying objects by touch: An "expert system." Perception \& Psychophysics, 37, 299-302.

Kramer, A. F., Tham, M.-P., \& Yeh, Y.-Y. (1991). Movement and focused attention: A failure to replicate. Perception \& Psychophysics, 50, 537-546.

KRUEGER, L. E. (1970). Effect of bracketing lines on speed of "same" "different" judgment of two adjacent letters. Journal of Experimental Psychology, 84, 324-330.

Krueger, L. E. (1978). A theory of perceptual matching. Psychological Review, 85, 278-304.

Lederman, S. J., \& Klatzky, R. L. (1987). Hand movements: A window into haptic object recognition. Cognitive Psychology, 19, 342-368.

Lederman, S. J., \& KLATZKY, R. L. (1990). Haptic exploration and object representation. In N. E. Gordale (Ed.), Vision and action: The control of grasping (pp. 98-108). Norwood, NJ: Ablex.

Mozer, M. C. (1989). Types and tokens in visual letter perception. Journal of Experimental Psychology: Human Perception \& Performance, 15, 287-303.

Nickerson, R. S. (1965). Response times for "same".-"different" judgments. Perceptual \& Motor Skills, 20, 15-18.

Proctor, R. W. (1981). A unified theory for matching-task phenomena. Psychological Review, 88, 291-326.

Sathian, K., Burton, H. (1991). The role of spatially selective attention in the tactile perception of texture. Perception \& Psychophysics, 50, 237-248.

Smith, L. B., \& Evans, P. M. (1989). Similarity, identity and dimensions: Perceptual classification in children and adults. In B. E. Shepp \& S. Ballesteros (Eds.), Object perception: Structure and process (pp. 325-356). Hillsdale, NJ: Erlbaum.

Treisman, A., Gelade, G. (1980). A feature integration theory of attention. Cognitive Psychology, 12, 97-136. 


\section{NOTES}

1. In the course of our preliminary experiments, we tested the index finger as a target site, as we had in previous studies (Evans \& Craig, 1991, 1992). The nontarget locations we used were either the middle finger, the adjacent site, or the little finger, the nonadjacent site. We found considerable interference with both nontarget sites; however, a comparison of the effectiveness of the two nontarget sites also showed that the middle finger, the adjacent site, produced significantly greater interference than the little finger, the nonadjacent site. Before concluding that distance per se was responsible for the decline in interference when the little finger was the nontarget site, we tested the two nontarget sites again, but with a target site adjacent to both, the ring finger. We found that both the middle and little finger sites produced significant interference in processing movement on the ring finger, but that significantly less interference was seen when the little finger was the nontarget site than when the middle finger was the nontarget site. This result strongly suggested that the little finger was simply less effective than the middle finger as a nontarget site.

2 . Both the accuracy and reaction-time trials in which the reaction times were less than or equal to $150 \mathrm{msec}$ or greater than $1,000 \mathrm{msec}$ were not included in the analysis. These trials accounted for less than $1 \%$ of the trials.

(Manuscript received January 2, 1992; revision accepted for publication May 11, 1992.) 